\title{
EI IMARPE y el número especial Ecología, pesquería y conservación del jurel (TRACHURUS MURPHYI) en el Perú
}

\section{The IMARPE and the special issue Ecology, fisheries and conservation of Jack mackerel (TRACHURUS MURPHYI) in Peru}

\section{Germán Vásquez Solís Talavera}

Instituto del Mar del Perú, esquina Gamarra y General Valle S/N Chucuito, Callao, Perú.

Email Germán Vásquez Solís Talavera:

presidencia@imarpe.gob.pe
Citación:

Vásquez Solís Talavera G. 2013. EI IMARPE y el número especial Ecología, pesquería y conservación del jurel (Trachurus murphyi) en el Perú. En: Csirke $\mathrm{J}$. R. Guevara-Carrasco \& M. Espino (Eds.). Ecología, pesquería y conservación del jurel (Trachurus murphyi) en el Perú. Rev. peru. biol. número especial 20(1): 003- 004 (Septiembre 2013)
El mar peruano es la cuarta región natural del Perú y es considerado como uno de los sistemas más productivos del planeta, si no el más. Su particular situación geográfica le permite contar con características muy propias que lo convierten en un inmenso sistema de producción de biomasa, que sustenta una compleja malla trófica de comunidades biológicas que se desarrollan en condiciones de variabilidad extrema.

Dentro de este escenario de alta complejidad, el mar peruano ha sido una fuente de alimento para el poblador peruano, desde tiempos inmemoriales. Así lo indican múltiples testimonios que se encuentran en restos arqueológicos de nuestras ancestrales culturas afincadas en la costa peruana desde hace más de 6000 ańos, tales como Caral, Bandurria y Áspero, donde se reportan diferentes especies de peces y moluscos, entre las cuales destaca el jurel como parte de la dieta de nuestros ancestros.

El Estado peruano inicia los estudios formales de los recursos del mar peruano desde fines del siglo XIX e inicios del siglo XX, en la época de la explotación del guano de isla, producto natural que marcó una importante etapa en la economía y la historia nacional. Luego, las investigaciones fueron continuadas por el Instituto del Mar del Perú (IMARPE) creado en 1964; una década en la cual el Perú comenzó a convertirse en uno de los países más importantes del mundo en materia de producción pesquera. Gracias a la actitud visionaria de las autoridades y actores principales de la pesca nacional de aquella época y al efectivo aporte de la cooperación internacional, el IMARPE pudo amalgamar un notable grupo de trabajo conformado por profesionales, técnicos y administrativos que han logrado constituir y mantener un importante sistema de investigación del mar peruano y sus pesquerías. Esto ha permitido que hoy en día el Perú cuente con un buen y creciente conocimiento sobre la compleja dinámica de su mar y sus recursos vivos, así como con una extraordinaria base de datos científicos, considerada por muchos expertos como una de las más extensas y completas del mundo. Esto se manifiesta en el creciente interés de entidades nacionales e internacionales por cooperar con el IMARPE en la investigación de un mar de características únicas.

Desde que asumí la Presidencia del Consejo Directivo del IMARPE en septiembre de 2011, pude constatar la complejidad de las labores que realizan sus científicos y de las dificultades que enfrentan con mucho profesionalismo para cumplir con su misión, muchas veces en condiciones muy difíciles generadas por las carencias propias de un país en desarrollo como el nuestro.

Gracias al denodado esfuerzo de quienes laboran y han laborado en el IMARPE desde su creación, es que hoy podemos contar con un acervo de conocimiento científico que respalda la toma de decisiones para la gestión del uso de los recursos vivos del mar. Dicho conocimiento científico se manifiesta en las publicaciones científicas periódicas que el IMARPE ha venido produciendo a lo largo de su historia en sus labores de evaluación y conocimiento de nuestros recursos marinos y que, desde este año 2013 tienen visibilidad y un uso abierto mayor a través del Repositorio Digital inserto en la página web institucional.
Publicado online: $\quad 11 / 10 / 2013$ Publicado impreso: 15/10/2013 
Además de las publicaciones periódicas, el IMARPE ha producido notables e importantes publicaciones científicas entre las que podríamos citar las siguientes: Atlas del Instituto del Mar del Perú (1965), Oceanografía de las aguas costeras del Perú (1970), Temperatura promedio de la superficie del mar frente a la costa peruana, periodo 1928-1969 (1972), Clave para identificar los peces marinos del Perú (1974, 1998), La investigación cooperativa de la Anchoveta y su Ecosistema (ICANE) entre Perú y Canadá (1981), El Niño y su impacto en la Fauna Marina (1985), Lista sistemática de moluscos marinos del Perú (1987), The Peruvian Anchoveta and its Upwelling Ecosystem: Three decades of Change (1987), Recursos y dinámica del ecosistema de afloramiento peruano (1988), The Peruvian Upwelling Ecosystem: Dynamics and Interactions (1989), Catálogo comentado de los peces marinos del Perú (2001), The Northern Humboldt Current System: Ocean Dynamics, Ecosystem Processes, and Fisheries (2008) y el Libro de peces de aguas profundas del Perú (2009).

Aprovechando la oportunidad que nos brinda la Universidad Nacional Mayor de San Marcos a través de la Revista Peruana de Biología, me corresponde la tarea de presentar un esfuerzo de síntesis sobre una de las especies emblemáticas del mar peruano: el jurel. Ésta es y ha sido una de las especies más importantes del consumo humano directo del poblador nacional desde épocas remotas, por lo cual desde el año 2002 sólo se le puede pescar para ese fin. Su situación ha sido objeto de múltiples controversias en los últimos años, en gran medida por el desconocimiento de su dinámica poblacional en relación a la naturaleza del mar peruano. Por ello, en un esfuerzo estrictamente institucional, 27 investigadores del IMARPE asumieron la tarea de elaborar una síntesis del conocimiento de esta especie, la misma que se presenta ahora como un avance en trece capítulos o artículos de este primer volumen del IMARPE y de la Revista Peruana de Biología dedicado enteramente al jurel.

Con esta obra titulada Ecología, pesquería y conservación del jurel (TRACHURUS MURPHYI) en el Perú, se pone a disposición del público nacional e internacional, más de cinco décadas de información sobre sus características biológicas y ecológicas, sobre su pesquería y la complejidad de su manejo, constituyéndose en un referente obligado para aquellos que quieran investigar y conocer sobre este importante recurso.

Antes de finalizar, debo destacar que coincidentemente la publicación de este trabajo científico se produce en el año del jubileo por la conmemoración del quincuagésimo aniversario de creación del IMARPE. Entendemos que esa es una buena señal, pues además hay otros esfuerzos internos en camino, que esperamos vean la luz a los 50 años de creación de una entidad de la cual los peruanos debemos sentirnos orgullosos. Al difundir el conocimiento científico sobre el mar peruano, también estamos cumpliendo con la misión de incorporar a más peruanos al torrente de ciudadanos informados que puedan tener una visión más completa y objetiva sobre la naturaleza de los recursos vivos marinos de esta nuestra todavía poco conocida cuarta región geográfica.

Finalmente, expreso mi agradecimiento personal y calurosa felicitación a todos los actores de dentro y fuera del IMARPE sin cuyo esfuerzo no habría sido posible esta magnífica obra.

Callao, setiembre de 2013.

Calm. (r) Germán Vásquez Solís Talavera

Presidente del Consejo Directivo del IMARPE

\section{Agradecimientos}

Jorge Csirke, Renato Guevara-Carrasco y Marco Espino, editores de este número especial de la Revista Peruana de Biología deseamos agradecer a los colaboradores y expertos nacionales y extranjeros, que han contribuido con sus valiosos comentarios, críticas y sugerencias a la realización de esta obra; en especial a: Arnaud Bertrand (Sète Cedex, Francia), Omar DeFeo (Montevideo, Uruguay), Francois Gerlotto (Castelnau-le-Lez, Francia), Jordi Lleonart (Barcelona, España), Takahisa Mituhasi (Tokyo, Japón), Orlando Mora (Bogotá, Colombia), Jorge Oliva (Trujillo, Perú) y Leo Walter González (Isla Margarita, Venezuela). También un especial reconocimiento a Pedro Trillo por su valiosa e incansable asistencia en las diversas fases de la producción de este volumen y al apoyo de Sara Bravo en la corrección ortográfica, Gaby Matsumoto en el cuidado de las figuras y Giancarlo Pinasco en algunas traducciones. Por último, queremos agradecer a la Universidad Nacional Mayor de San Marcos por habernos acogido como editores y por todo el apoyo brindado en la producción de este número especial. 